\title{
Introduction
}

Molecularly-

Mediated

\section{Assembly of Gold Nanoparticles}

\author{
I-Im S. Lim and Chuan-Jian Zhong* \\ Department of Chemistry, State University of New York \\ at Binghamton, Binghamton, New York 13902 \\ ${ }^{*}$ To whom correspondence should be addressed. \\ e-mail: cjzhong@binghamton.edu
}

\begin{abstract}
.
The understanding of the interparticle interactions and reactivities is essential to the exploitation of the unique optical, electronic, and chemical or biological properties of gold nanoparticles in many areas of nanotechnology. This paper describes findings of a comparison of optical properties of molecularlymediated assemblies of gold nanoparticles where the interparticle interactions and spatial properties are defined by molecular mediators and templates. The changes of the surface plasmon resonance band of the assemblies of gold nanoparticles mediated by several different types of molecular mediators, including multidendate methylthiosilane thioether, homocysteine, 1-(4-methyl)-piperazinyl fullerene, and indolenine cyanine dye, are compared. In the assembly process, the size of individual $\mathrm{Au}$ nanoparticles and the volume fraction of gold nanoparticles in the solution should remain largely constant, whereas the interparticle distance changes, which leads to changes in the interparticle dielectric medium constant or refractive index. The changes in the SP band in relation to the change in effective refractive index are discussed, along with their implications to assembly sizes, interparticle interactions, and potential applications in designing electrical and optical sensors.
\end{abstract}

Gold nanoparticles have attracted a wide range of interest due to increasing applications in sensors, biosensors, medicine, catalysis and many emerging areas of nanotechnology. These applications require the ability to control the size, shape, surface, and interparticle properties of gold nanoparticles. While the synthesis of gold nanoparticles of various sizes has been well documented, the ability to engineer gold nanoparticles as nanobuilding blocks toward functional architetures is increasingly challenging. This is especially true in those applications where the assembly of nanoparticles plays an important role in the optical, electronic, or spectroscopic properties, as demonstrated in many examples of assemblies created by chemical or biological molecules such as DNA 1 , polymer or dendrimer ${ }^{2-3}$, functionalized thiols $s^{4-5}$, multidentate thioethers ${ }^{6}$, multicharged fullerenes ${ }^{7}$, and charged dyes ${ }^{8}$.

In many of these assemblies, one of the most important signatures for assessing the interactions and reactivities is the change of the surface plasmon (SP) resonance band of gold nanoparticles. The origin of the SP band is the coherent excitation of free conduction electrons on the nanoparticle surface as the electromagnetic wave interacts with the nanoparticle surface. The oscillation of the electrons creates a change in the electronic dipole moment that can be detected in the UV-Visible region. The wavelength of this band depends on size, shape, and interparticle dielectric medium properties. When gold nanoparticles are assembled into close proximity, the SP band shifts as a result of interparticle dipole interactions or dielectric medium changes. In Mie theory ${ }^{9-10}$, the extinction coefficient is related to the volume concentration of the particles (individual or assembled nanoparticles), the interparticle distance, and the dielectric constant or the refractive index (RI) in the surrounding medium ${ }^{6,9-10}$. If the refractive index changes, the SP band will change in both wavelength and absorbance. There have been a number of theoretical studies delineating the effect of RI or dielectric medium properties with the $\mathrm{SP}$ band in systems involving interactions between metal nanoparticles ${ }^{11-13}$. Recently, the study of how the plasmon resonance wavelength of metal nanoparticles is affected by varying RI of the surrounding medium showed that upon hybridization in a DNA-nanoparticle assembly, the plasmon band shift increases linearly with the change of $\mathrm{RI}^{12}$. The study of the shift of the $\lambda_{\max }$ upon the adsorption of alkanethiol on triangular Ag nanoparticles showed that there is a red shift of $3 \mathrm{~nm}$ for every additional carbon atom in the alkane chain, whereas the Ag-S bond shows a blue shift due to the charge transfer interaction ${ }^{13}$. While the shift of the SP band could be modeled by either the change in RI or the dipole-dipole interaction, or both, the consideration of the change in RI is one of the important ways to assess the SP band shift. The dependence of the SP band on the interparticle medium properties has indeed been established for several systems. However, the correlation of the optical properties with 


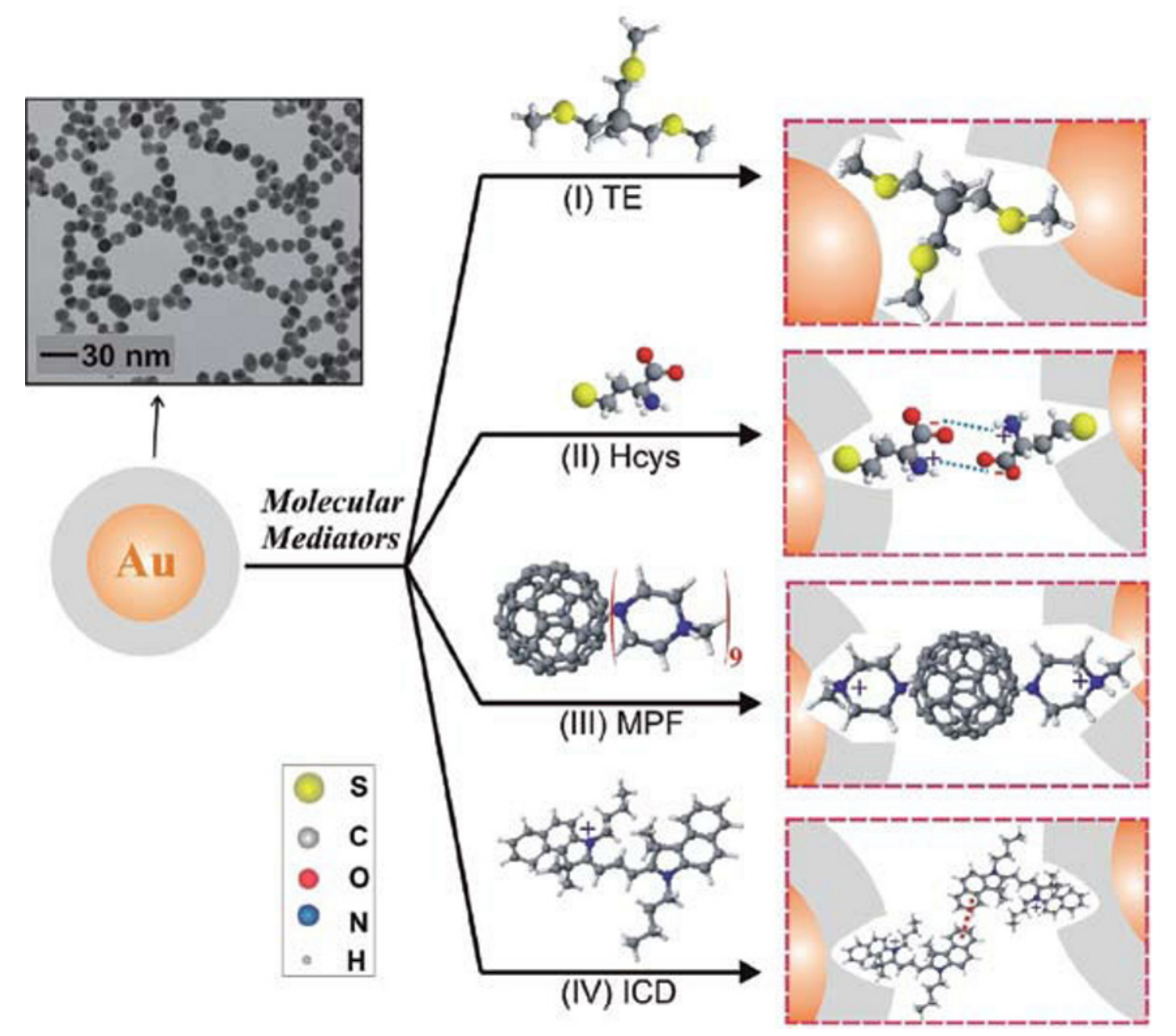

\section{Scheme 1}

Schematic illustration of molecularly-mediated assemblies and interparticle interactions. (I) multidendate methylthiosilane thioether (TE), (II) homocysteine (Hcys), (III) 1-(4-methyl)-piperazinyl fullerene (MPF), and (IV) indolenine cyanine dye (ICD). Insert: TEM image for 11-nm gold nanoparticles.

different assemblies of gold nanoparticles remains elusive.

In this paper, we focus on the SP band changes for molecularly-mediated assemblies of gold nanoparticles where the interparticle interactions and spatial properties are defined by molecular mediators and templates. In particular, we examined how the SP band change is related to the dielectric medium properties or refractive index in the assembly of gold nanoparticles mediated by several types of molecules, including (I) multidendate methylthiosilane thioether (TE), (II) homocysteine (Hcys), (III) 1-(4-Methyl)piperazinyl fullerene (MPF), and (IV) indolenine cyanine dye (ICD). The molecular structures of these mediators and their interparticle interactions are illustrated in Scheme 1. In each system, the changes in the SP band in relation to the change in RI will be discussed, along with discussions of the specific interparticle interactions or reactivity.

\section{Experimental Section}

Hydrogen tetrachloroaurate $\left(\mathrm{HAuCl}_{4}, 99 \%\right)$, sodium citrate ( $\mathrm{Na}_{3} \mathrm{Cit}, 99 \%$ ), sodium chloride ( $\left.\mathrm{NaCl}, 99+\%\right)$, homocysteine (Hcys, 95\%), tetraoctylammonium bromide ( $\left.\mathrm{TOA}^{+} \mathrm{Br}^{-}, 99 \%\right)$ and sodium borohydride $\left(\mathrm{NaBH}_{4}, 99 \%\right)$ were purchased from Aldrich and used as received. Water was purified with a Millipore Milli-Q water system. The synthesis of met hyltris[(methylthio)methyl]silane $\left(\mathrm{MeSi}\left(\mathrm{CH}_{2} \mathrm{SCH}_{3}\right)_{3}, \mathrm{TE}\right)^{14}$ and 1-(4-methyl)-piperazinyl fullerene (MPF) ${ }^{15}$ was described previously. Cyanine dyes were obtained from Crysta-lyn Chemical Inc.

The synthesis of citrate-capped aqueous soluble gold nanoparticles followed the reported procedure $^{16}$, and details of our synthesis procedures were described previously ${ }^{8,17}$. Theaveragesize of thenanoparticles determined by transmission electron microscopy was $11.5 \pm 0.6 \mathrm{~nm}$. The synthesis of the organic soluble gold nanoparticles capped with monolayer shells of tetraoctylammonium bromide were synthesized using the two-phase protocol. Details of the synthesis procedures were described previously ${ }^{6}$. The 
particles have an average diameter of $5.1 \pm 0.9 \mathrm{~nm}$.

UV-Visible (UV-Vis) spectra were acquired with an HP 8453 spectrophotometer. Spectra were collected over the range of 200-1100 nm. In a typical experiment, the stock solution of nanoparticles was diluted to reach a final concentration in the $\mathrm{nM}$ range for the $11-\mathrm{nm}$ nanoparticles and $\mu \mathrm{M}$ range for the 5-nm nanoparticles. Next, a quantitative amount of mediators (e.g., TE, Hcys, MPF or ICD) was added into the nanoparticle solution followed by quick agitation to mix the solution, and UV-Vis spectral evolution was followed. The experiment was conducted under ambient conditions. In the spectral simulation, refractive indices of 1.25 and 1.46 were used for the initial aqueous and toluene solutions of gold nanoparticles, respectively.

\section{Results and Discussion}

\section{$1 \quad$ Multidentate Methylthiosilane Thioether- Mediated Assembly}

The methylthiosilane thioether mediated assembly of gold nanoparticles (5-nm) into spheres of controllable sizes in organic solution serves as an excellent model system to delineate the various parameters in the SP band evolution. This system involves a mediator-template assembly in which the multidentate thioether molecules act as the mediators and the capping agent tetraalkylammonium bromide $\left(\mathrm{TOA}^{+} \mathrm{Br}^{-}\right)$provides the templating effect ${ }^{6}$. The nanoparticles form spherical assemblies via the coordination bonding of thioether groups, involving 1) bridging of the nanoparticles by the mediator, and 2) templating force exerted by the TOA capping molecules. For example, the addition of TE into a toluene solution of TOA-capped Au nanoparticles $\left[\mathrm{Au}_{\mathrm{nm}}\right](0.1 \mu \mathrm{M})$ leads to the observations of spherical assemblies of nanoparticles and the associated longwavelength SP band (Figure 1a-b). As the ratio of [TE] / [Au $\left.\mathrm{Am}_{\mathrm{nm}}\right]$ (r) is decreased (from 2000, 200, 10 and 1), the SP band shows a red shift (b), which is a result of the increased size of the spherical assembly as evidenced by TEM (a).
It is important to note that the $r$-dependent change of the diameter for the dried spherical assemblies of nanoparticles determined from TEM data showed a trend similar to the change in the hydrodynamic diameter determined from dynamic light scattering (DLS) measurements (Figure 1a). The fact that the DLS-determined sizes are larger than the TEM-determined sizes reflects the presence of solvent and ions around the spherical assemblies in the solution. For the SP band, the position for the long-wavelength band $\left(\lambda_{\max }\right)$ displays an approximate linear relationship with the average diameter of the spherical assembly (Figure 1b). It is necessary to point out that we described previously some related experimental results for this type of nanoparticle assembly ${ }^{6}$. The focus of the present paper deals with the comparison of the optical properties of many different types of nanoparticle assembly by spectral simulation based on Mie theory, focusing mainly on how the shift of the SP band for the different assemblies can be correlated to the change in the refractive index (RI). For $\Delta \mathrm{d}_{\text {sphere }}=1 \mathrm{~nm}$, there was about an increase of $1.3 \mathrm{~nm}$ in wavelength. Assuming a layer-bylayer assembly in the sphere, each additional nanoparticle layer would correspond to an increase of $\sim 7 \mathrm{~nm}^{6}$, which would then lead to an increase of $\sim 9 \mathrm{~nm}$ in wavelength. On the basis of this observation, Mie theory was applied to the analysis of the SP band evolution. In our earlier work ${ }^{6}$, we showed a spectral simulation based on Mie theory (eqn. 1), ${ }^{9-10}$

$$
\frac{\gamma}{N V}=\frac{18 \pi \varepsilon_{\alpha}^{3 / 2}}{\lambda} \times \frac{\varepsilon_{2}}{\left(\varepsilon_{1}+2 \varepsilon_{\alpha}\right)^{2}+\varepsilon_{2}^{2}}
$$

$(\gamma$. extinction coefficient, $N V$ : volume concentration of the assembled nanoparticles ( $\mathrm{N}$ : the number of spheres per unit volume; $\mathrm{V}$ : the volume of each sphere), $\varepsilon_{\alpha}$ : dielectric medium constant, $\varepsilon_{1}$ and $\varepsilon_{2}$ : the real and complex part of $\mathrm{Au}$ ), and a modified Mie theory ${ }^{10}$. It was shown that the dielectric medium constant increased by a factor of $\sim 5$ from individual nanoparticles to spherical assemblies, reflecting the difference in their interparticle dielectric medium
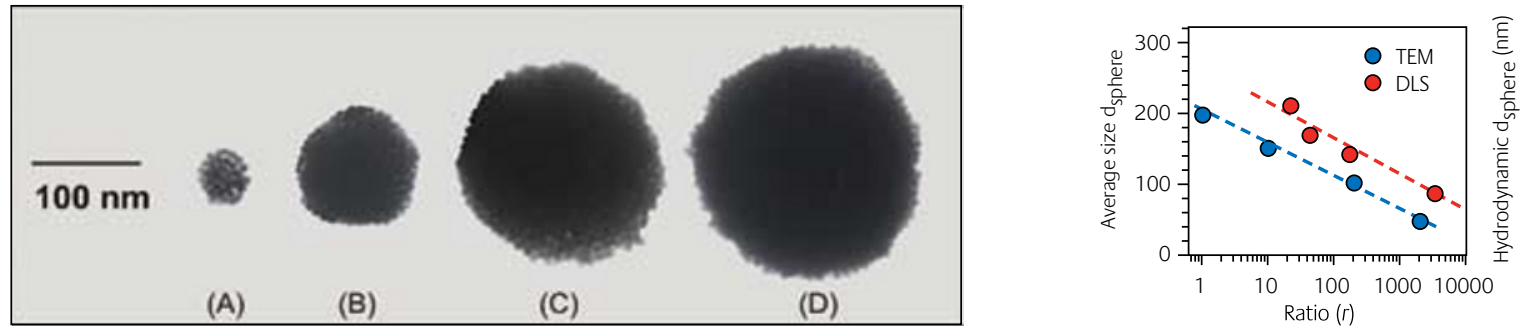

Figure 1 a

(a) Size features for the spherical assemblies of gold nanoparticles as a function of [TE]/[Au $\left.u_{n m}\right]$ ratio (r). (b) SP band for Au nanoparticles $(5-n m)(r e d)$ and TE-mediated spherical assemblies of the nanoparticles, and the average diameter of the spherical assembly vs. $\lambda_{\max }$ of the longwavelength SP band. (c) Simulation results for the SP band of Au particles (red) and the long-wavelength SP bands in b, and the change of refractive index $(\Delta n)$ used in the simulation vs. $\lambda_{\max }$. 

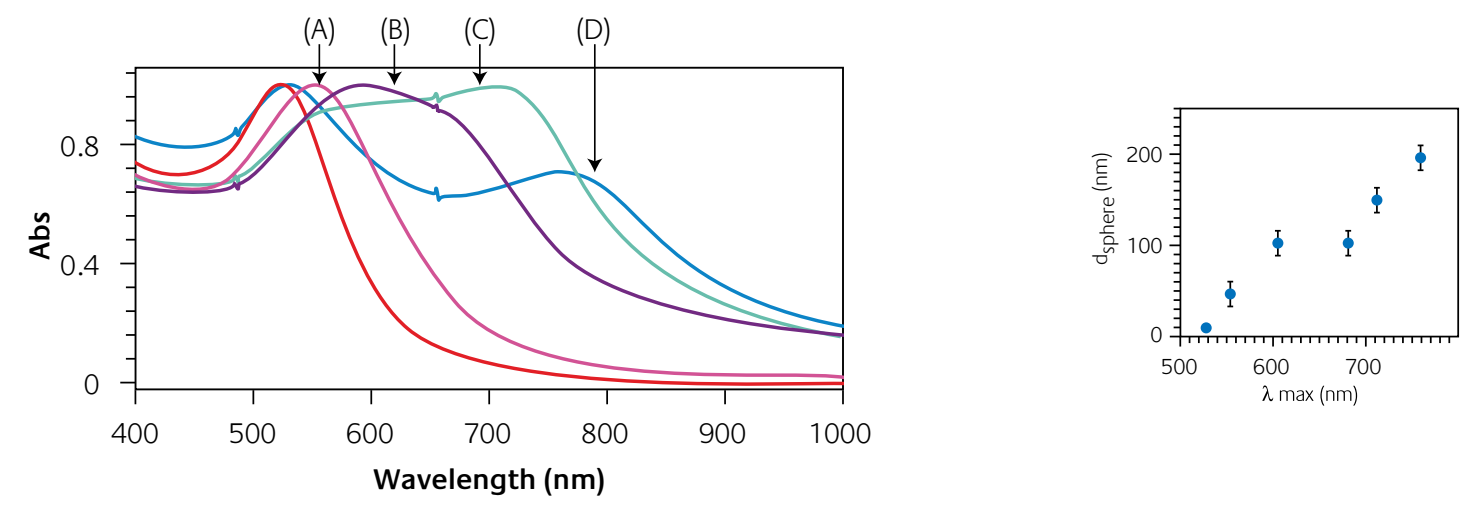

Figure $1 \mathrm{~b}$
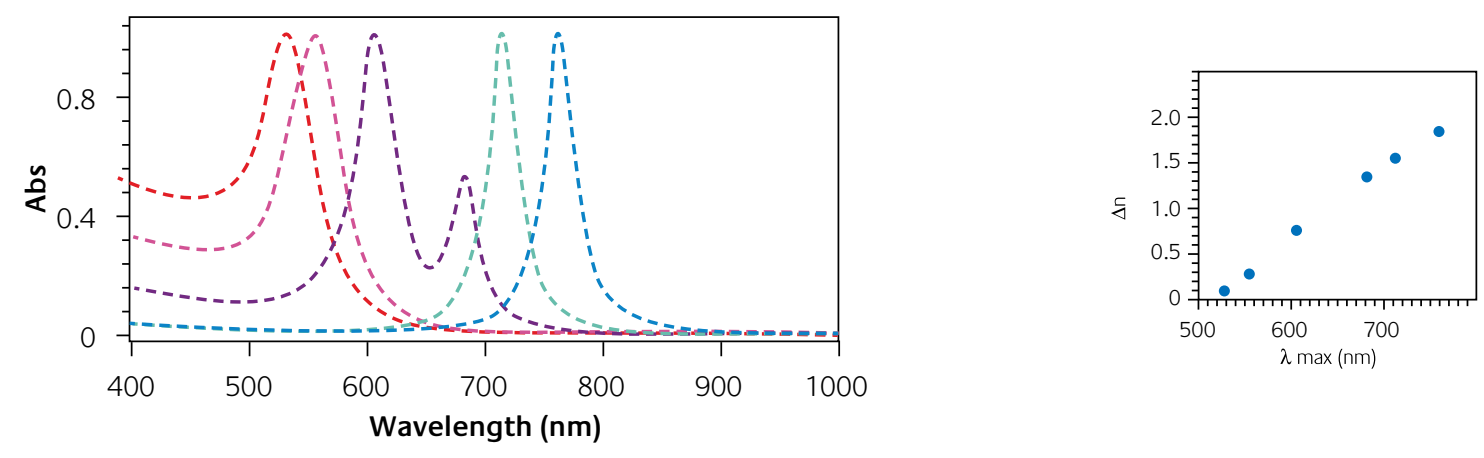

Figure $1 \mathrm{c}$

properties. The observation of an isosbestic point between the short- and long-wavelength bands implies the presence of two distinct light-absorbing species in the solution, corresponding to individual nanoparticles and spherical assemblies of the nanoparticles. In comparison with $\varepsilon=$ 1.9 obtained for the individual Au nanoparticles, values of $\varepsilon=3.5$ and 9.4 were found for 525-nm and 710-nm bands, respectively, in the spectrum for the spherical assemblies of nanoparticles. This finding is indicative of changes in interparticle distances and dielectric medium properties. Moreover, by spectral deconvolution and peak integration, we were able to determine the thermodynamic factor governing the mediator-template assembly for the spherical assemblies in solution ${ }^{6 \mathrm{~d}}$. The mediator-template assembly was shown to be an enthalpy-driven process in which the enthalpy change $(-1.3 \mathrm{kcal} / \mathrm{mole})$ is close to the magnitude of van der Waals interaction energy for alkyl chains and the condensation energy of hydrocarbons.

Since the dielectric medium constant $(\varepsilon)$ is related to refractive index, i.e., $\left(n_{\text {eff }}+i k_{\text {eff }}\right)^{2}=\varepsilon_{\text {eff }}\left(\varepsilon_{\text {eff }}\right.$ is the effective dielectric constant of the nanocomposite ${ }^{10,18}, n_{\text {eff }}$ is the real part of the effective complex index of refraction for the nanocomposite, $k_{\text {eff }}$ is the imaginary part of the effective complex index of refraction), we show here that the evolution of the SP band at the longer wavelength region can be considered a result of the increase in effective refractive index for nanoparticles in the assembly (Figure 1c). In the assembly process, the size of individual Au nanoparticles and the volume fraction of gold nanoparticles in the solution should remain largely constant, whereas the interparticle distance changes, which leads to changes in the interparticle dielectric medium constant or refractive index. Refractive index $n$ will be used to represent the $n_{\text {eff }}$ throughout this report. Interestingly, the change in $\mathrm{n}$ was found to exhibit an approximate linear relationship with $\lambda_{\max }$ (Figure 1c). The plot of such fitting results provides one way to assess the correlation of the change in assembly size with the optical properties, though is not necessarily the only way. For $\Delta n$ $=0.1$, an increase of $13 \mathrm{~nm}$ in wavelength was determined. While this increase is in qualitative agreement with findings in other studies on the effect of RI on SP band in systems involving interactions between gold nanoparticles (e.g., for dimer pairs of gold nanoparticles (12-nm size), $\Delta \lambda=6.2 \mathrm{~nm}$ was reported for $\left.\Delta n=0.1^{12}\right)$. The subtle difference between nanoparticles in our large spherical assemblies vs. in dimers is not surprising in view of the difference in local environment of the nanoparticles. It is well demonstrated for polymers in solvent that the change in refractive index is proportional to the concentration. 


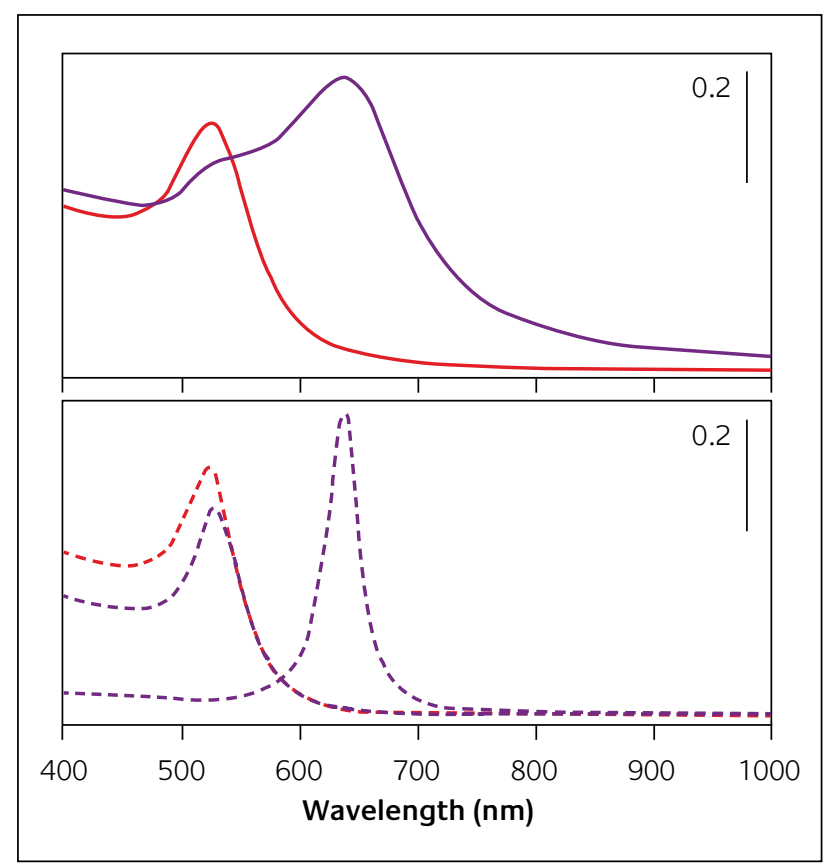

\section{Figure 2}

Spectra comparing the Au nanoparticles of 11-nm diameter (red) and the Hcys mediated assembly of the particles (blue) in aqueous solution. Bottom panel: Simulation results of the SP band of the 11-nm Au particles and the assembly of the particles in water. ( $\Delta n=0.11$ and 1.20 were used for $525 \mathrm{~nm}$ and $635 \mathrm{~nm}$ bands (blue dashed lines)).

\section{Homocysteine-Mediated Assembly of Nanoparticles}

Homocysteine (Hcys), a thiol containing amino acid, is another intriguing mediator for the assembly of gold nanoparticles in aqueous solution ${ }^{19}$. The presence of Hcys in human blood is considered to be an important risk factor in medical diagnostics for many different diseases. For the assembly of gold nanoparticles, the Hcys-gold nanoparticle interaction involves 1) the adsorption of Hcys on the nanoparticle surface, displacing the original citrate-capping molecules and 2) the zwitterion-type electrostatic interactions between the Hcys groups bound to the gold nanoparticle surface. The changes in interparticle distance as a result of the interparticle interaction lead to the shift in the SP band.

Upon addition of Hcys to an aqueous solution of 11-nm sized Au nanoparticles (e.g., [Au particles] $=2.3 \mathrm{nM}$; [Hcys] $=9.2 \mu \mathrm{M})$, the absorbance at $\sim 520 \mathrm{~nm}$ decreases while a longer-wavelength band ( $\sim 635 \mathrm{~nm})$ evolves (Figure 2, solid line). This evolution reflects the decrease of the isolated gold nanoparticles and the formation of the Hcys-mediated assembly of nanoparticles in the solution.

Figure 2 also shows the simulation result for an assembly of 11-nm particles (dashed line) using Mie theory ${ }^{6 a}{ }^{10}$, which matches with the bands in terms of the SP wavelength as observed (solid line). On the basis of the documented principle that the plasmon red shift $(\Delta \lambda)$ increases with refractive index change $(\Delta n)$, a basic assumption for applying the Mie theory simulation to this system is that the nanoparticles within the assembly environment have a refractive index higher than that in the water environment. This assumption

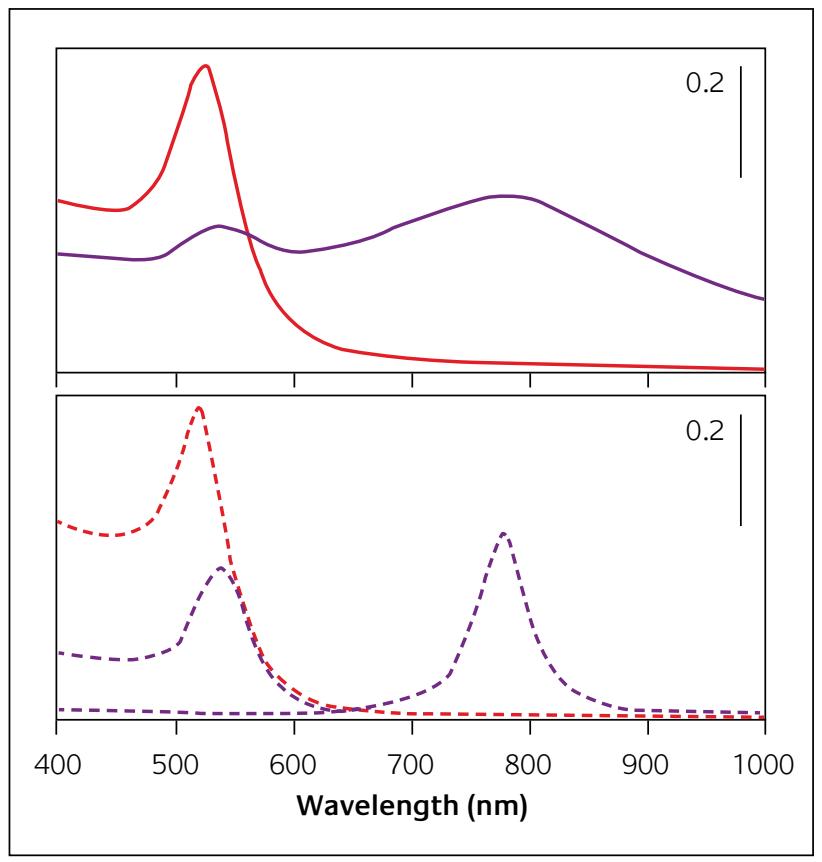

\section{Figure 3}

Spectra comparing the Au nanoparticles of 30-nm diameter (red) and the Hcys mediated assembly of the particles (blue) in aqueous solution. Bottom panel: Simulation results for the SP band of the 30-nm Au particles and the assembly of the particles in water ( $\Delta n=0.39$ and 1.93 were used for $535 \mathrm{~nm}$ and $785 \mathrm{~nm}$ bands (blue dashed lines)).

is qualitatively supported by the trend of refractive index changes reported in previous studies for similar assemblies of metal nanoparticles in different systems ${ }^{20}$. The simulation result yielded $\Delta n=1.2$ for the long-wavelength SP band. In view of the red shift of $115 \mathrm{~nm}$ in wavelength of this band in comparison with the initial SP band of Au nanoparticles, a change of refractive index $\Delta n=0.1$ translates to an increase of $9.6 \mathrm{~nm}$ in wavelength.

Similar spectral features were also observed when larger sized nanoparticles were used for the Hcys-mediated assembly. For example, the Hcys-mediated assembly of 30-nm sized $\mathrm{Au}$ nanoparticles in an aqueous solution of $\left[\mathrm{Au}_{30 \mathrm{~nm}}\right]=0.14 \mathrm{nM}$ and $[\mathrm{Hcys}]=7.8 \mu \mathrm{M}$ (Figure 3, solid line) displays a broader SP band with a greater shift to the longer wavelength region $(\sim 785 \mathrm{~nm})$. This shift may be due to the fact that the larger particles have a greater coupling tendency, resulting in a larger polarization of the surface electrons and stronger interparticle interactions.

The simulation result (dashed line) yielded $\Delta n=1.9$ for the long-wavelength SP band. In view of the $260 \mathrm{~nm}$ red shift in wavelength, the change of $\Delta n=0.1$ translates to an increase of $13.5 \mathrm{~nm}$ in wavelength. Note that there was also a subtle shift in the shorter-wavelength band, the simulation of which yielded $\Delta n=0.4$.

\section{1-(4-methyl)piperazinyl Fullerene Mediated Assembly of Nanoparticles}

Fullerenes serve as an important class of supramolecules for the assembly of nanoparticles which is expected to lead to interesting properties due to their well-defined sizes and 


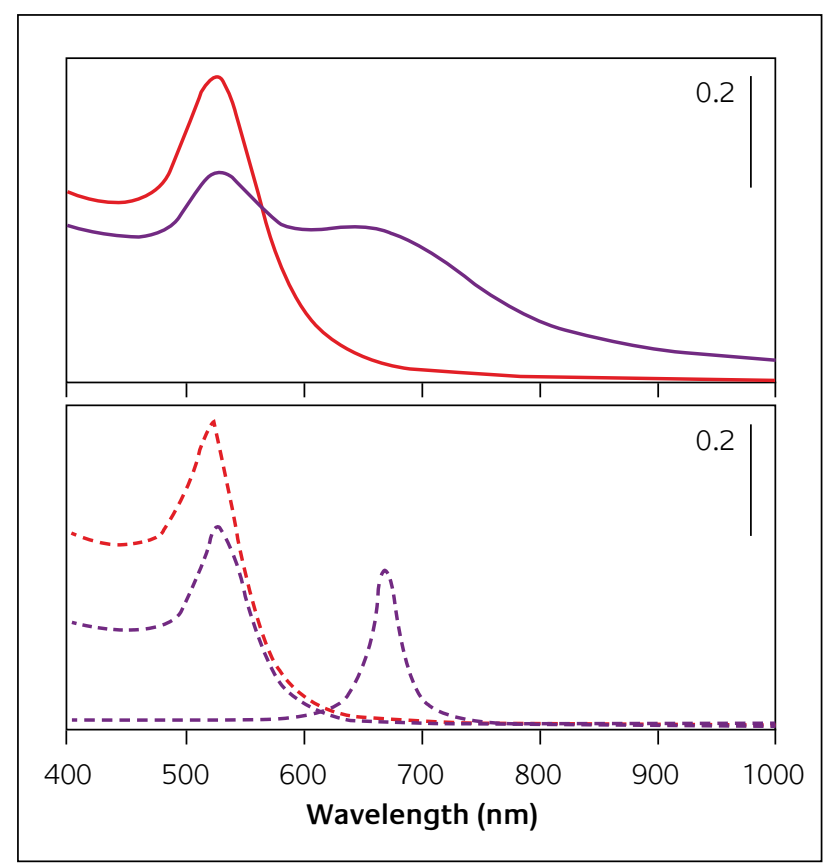

\section{Figure 4}

Spectra comparing the Au nanoparticles of 11-nm diameter (red) and the MPF-mediated assembly of the particles (blue) in aqueous solution. Bottom panel: Simulation results for the SP band of the 11-nm Au particles and the assembly of the particles in water $(\Delta n=0.15$ and 1.40 were used for $525 \mathrm{~nm}$ and $660 \mathrm{~nm}$ bands (blue dashed lines)).

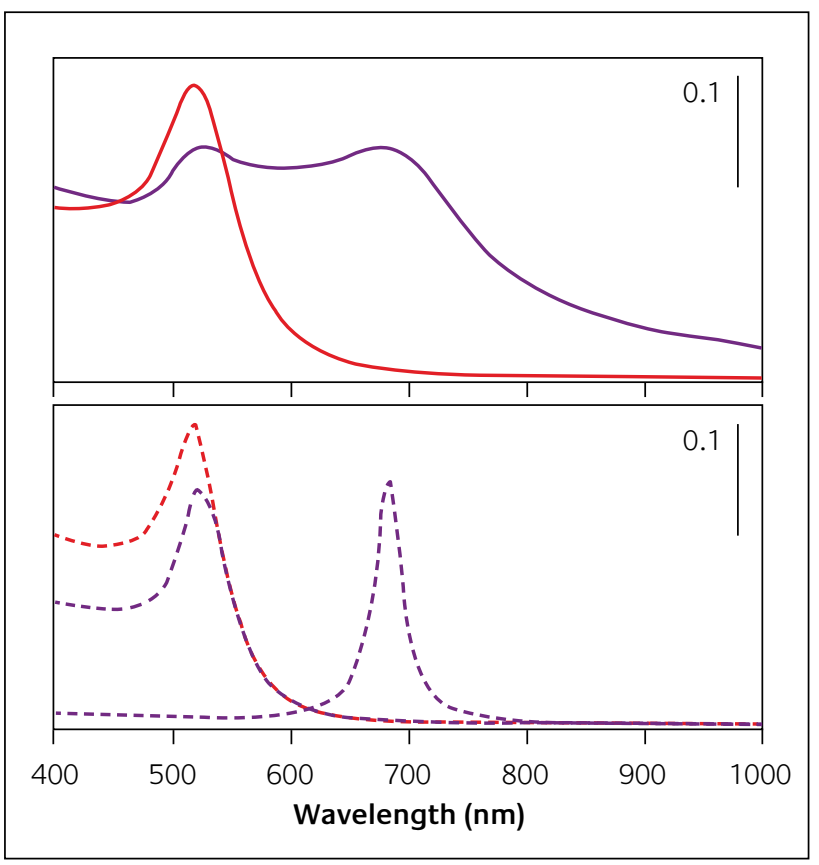

\section{Figure 5}

Spectra comparing the Au nanoparticles of 11-nm diameter (red) and the ICD mediated assembly of the particles (blue) in aqueous solution. Bottom panel: Simulation results for the SP band of the 11-nm Au particles and the assembly of the particles in water $(\Delta n=0.15$ and 1.53 were used for $525 \mathrm{~nm}$ and $685 \mathrm{~nm}$ bands (blue dashed lines)).

shapes with potential applications involving optical sensing, electron/energy transfer, controlled drug delivery, etc. We investigated the viability of fullerenes with multifunctional binding sites as mediators for the assembly of gold nanoparticles in aqueous solutions? ${ }^{7}$. One example is 1-(4-methyl)piperazinyl fullerene (MPF), which contains nine positively-charged piperazinyl groups surrounding $C_{60}$. This system involves chemically tunable electrostatic interactions between the negatively-charged gold nanoparticles and the positively-charged MPF. In addition to TEM data, both DLS and SERS data confirmed the assembly of nanoparticles into large clusters and the adsorption of MPF on the gold surface. Furthermore, the interparticle interactions between the supramolecules and gold nanoparticles were found to be $\mathrm{pH}$ tunable, leading to controllable assembly and disassembly by simply manipulating the solution $\mathrm{pH}$.

Figure 4 shows a representative set of spectra for the MPFmediated assembly of Au nanoparticles in an aqueous solution of $[$ Au particles] $=1.70 \mathrm{nM}$ and $[\mathrm{MPF}]=6.89 \mu \mathrm{M}$. The longwavelength SP band was found at $\sim 660 \mathrm{~nm}$ (solid line) upon the addition of MPF to the 11-nm sized Au nanoparticles. The spectral evolution is indicative of the change in interparticle distance and dielectric medium properties as a result of the assembly. A distinct isosbestic point is observed around $~ 580$ $\mathrm{nm}$, indicating the presence of two light-absorbing species at equilibrium, i.e., Au nanoparticles and MPF- Au assemblies.

The simulation result (dashed line) yielded $\Delta n=1.4$ for the long-wavelength SP band. In view of the red shift of 140 $\mathrm{nm}$ in wavelength, the change of $\Delta \mathrm{n}=0.1$ translates to an increase of $10.0 \mathrm{~nm}$ in wavelength.

\section{$4 \quad$ Indolenine Cyanine Dye Mediated Assembly of Nanoparticles}

Cyanine dyes were shown to mediate the assembly of gold nanoparticles in aqueous solution via a combination of interparticle electrostatic and $\pi-\pi$ stacking interactions ${ }^{8}$. This class of dye is fluorescent. The study of the immobilization of fluorescent dye molecules onto metal nanoparticles has attracted much attention for potential applications in chemical and biological systems. The interactions can be broken down into a two-step process: 1) the rapid adsorption of the dyes on the nanoparticle surfaces, and 2) the subsequent assembly of the nanoparticles via J-aggregation and hydrophobic interactions of the adsorbed dyes. The interparticle reactivity was shown to produce fluorescence quenching of the dyes along with the changes in the SP band of the nanoparticles. The spectral evolution for the J-band from the dye and the SP band of the particles is dependent on the chemical environment and the structure of the dyes.

The indolenine cyanine dye (ICD) mediated assembly of $11-n m$ sized Au nanoparticles serves as a representative example of the spectral evolution (Figure 5). Upon addition of ICDs into the solution ([Au particles] $=2.0 \mathrm{nM}$ and $[$ ICD] $=$ $0.33 \mu \mathrm{M})$, a new band emerges at a longer wavelength $\sim 685$ $\mathrm{nm}$ (solid lines). An isosbestic point is observed at $\sim 550$ $\mathrm{nm}$, indicating a reaction equilibrium involving two species (reactant and product). The wavelength for the new bands is strongly dependent on the concentration of the dye. The higher the dye concentration is, the longer the wavelength of the new bands becomes. 
Table 1

A comparison of SP band properties for different systems.

\begin{tabular}{lccccc}
\hline Mediators & $\begin{array}{c}\text { Avg. size of } \\
\text { Nanoparticles }\end{array}$ & $\begin{array}{c}\text { SP band of } \\
\text { nanoparticles }\end{array}$ & $\begin{array}{c}\text { SP band of } \\
\text { assembly }\end{array}$ & $\Delta \mathbf{n}$ & $\begin{array}{c}\Delta \boldsymbol{\lambda} \\
\text { for } \boldsymbol{\Delta}=\mathbf{0 . 1}\end{array}$ \\
\hline TE & 5 & 527 & $553-759$ & $0.23-1.74$ & $11.3-13.3$ \\
Hcys & 11 & 520 & 635 & 1.2 & 9.6 \\
Hcys & 30 & 525 & 785 & 1.9 & 13.5 \\
MPF & 11 & 520 & 660 & 1.4 & 10.0 \\
ICD & 11 & 520 & & 685 & 1.5 \\
\hline
\end{tabular}

The simulation result (dashed line) yielded $\Delta n=1.5$ for the long-wavelength SP band. In view of the $165 \mathrm{~nm}$ wavelength red shift, a change of $\Delta n=0.1$ translates to an increase of $10.8 \mathrm{~nm}$ in wavelength.

\section{Summary}

Table 1 shows a summary of the optical properties comparing the above assemblies mediated by four different types of molecules. In the case of the TE-mediated assembly of 5-nm Au nanoparticles in toluene solution, different values of $\Delta n$ are obtained ranging from 0.23 to 1.74 , reflecting the red shift of the SP band for each specific size of the spherical assembly with different $r$. For every $\Delta n=0.1$ in this system, the red shift in wavelength, $\Delta \lambda$ was found to change, ranging from 11.3 to $13.3 \mathrm{~nm}$ depending on the assembly size. When comparing the shift for the assemblies of 11-nm Au nanoparticles mediated by Hcys, MPF and ICD in the aqueous solutions, the values of the red shift were found to be remarkably similar. For every $\Delta n=0.1, \Delta \lambda$ was found to be $9.6,10.0$ and 10.8 for Hcys, MPF and ICD, respectively. $\Delta \mathrm{n}$ is clearly correlated with the red shift of the SP band for the nanoparticle assembly. For the Hcys-mediated assembly of 30-nm Au nanoparticles in aqueous solution, $\Delta \lambda=13.5$ $\mathrm{nm}$ was found for every $\Delta \mathrm{n}=0.1$. This value is greater than that found for the Hcys-mediated assembly of 11-nm Au nanoparticles in aqueous solution ( $\Delta \lambda=9.6$ for $\Delta n=0.1$ ), but is remarkably close to the larger-sized spherical assembly of 5-nm Au nanoparticles mediated by TE in the organic solution $(\Delta \lambda=13.3$ for $\Delta n=0.1)$. There is a correlation between $\Delta n$ and the size of the nanoparticle assembly. It is true that the shift of the SP band could be due to, or modeled by, either the change in RI or the dipole-dipole interaction, or both, we focused on the correlation of SP shift with RI change in this paper as one way to compare optical properties of the different assemblies, though this is not necessarily the only way. Part of our on-going work is looking into other theoretical models (i.e., dipole-dipole, effective medium theory, etc. $)^{21}$ to relate the shift of the SP band, which will be reported in the future.

In conclusion, the analysis of the SP bands of the molecularly-mediated assembly of $\mathrm{Au}$ nanoparticles has provided insight into the interparticle interactions and resulting optical properties. Such insights are useful for exploiting the nanostructured gold materials for the design of electrical and optical sensors. For example, the shift of the SP band position and intensity of the nanoparticle assembly can be related to the change in dielectric medium or RI properties, which provides a means to produce optical signals for uptaking or releasing of molecular species in the nanoparticle assembly. One specific example is the amino acid-nanoparticle assembly ${ }^{19}$, which was shown to disassemble upon uptaking or releasing in the nanoparticle assembly, producing the shift of the SP band as a result of the change in the dielectric medium or refractive indices. This type of interparticle property also has potential applications in controlled drug delivery. In regard to the exploitation of these nanomaterials for application in electrical sensors, the electrical response of nanoparticle assemblies to vapor sorption $^{22}$ serve as an excellent example illustrating the correlation of the electrical properties with interparticle dielectric medium properties. The understanding of the precise control of the interparticle properties will benefit these nanostructured sensing applications.

\section{Acknowledgement}

This work is supported by the National Science Foundation (CHE 0349040). Stephanie Lim acknowledges support of the National Science Foundation Graduate Research Fellowship. Authors also thank Drs. D. Rabinovich, T. Liu, S. Zhou, F. Goroleski, P. Njoki and J. Luo for their help in parts of the work.

\section{About the Authors}

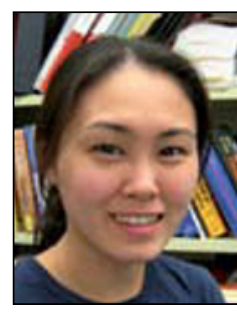

Stephanie Lim is a third year Ph.D. student at State University of New York at Binghamton, USA. Working in the area of nanoscience and nanotechnology under the direction of Dr. C.J. Zhong, part of her thesis research focuses on the molecularly mediated assembly of nano gold toward 
functional nanostructures for chemical/biological sensing and medical diagnostics. She is a recipient of the National Science Foundation Graduate Research Fellowship Award. For her nano gold research, she received a Best Poster Award at Gold 2006, Limerick, Ireland.

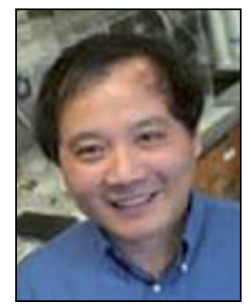

C.J. Zhong is an associate professor of chemistry at State University of New York. His research group works in the interdisciplinary fields of analytical, materials, catalysis, electrochemistry and nanoscience, and develops advanced nanomaterials for fuel cell, sensor and biomedical technologies.

\section{References}

1 (a) C.A. Mirkin, R.L. Letsinger, R.C. Mucic, JJ. Storhoff, Nature, 1996, 382, 607. (b) R. Elghanian, J.J. Storhoff, R.C. Mucic, R.L. Letsinger, C.A. Mirkin, Science, 1997, 277, 1078. (c) T.A. Taton, R.C. Mucic, C.A. Mirkin, R.L. Letsinger, J. Am. Chem. Soc, 2000, 122, 6305

2 (a) A.K. Boal, F. llhan, J.E. DeRouchey, T. Thurn-Albrecht, T.P. Russell, V.M. Rotello, Nature, 2000, 404, 746. (b) A.K. Boal, V.M. Rotello, J. Am. Chem. Soc, 2002, 124, 5019. (c) B.L. Frankamp, A.K. Boal, V.M. Rotello, J. Am. Chem. Soc, 2002, 124, 15146

3 N.N. Kariuki, L. Han, N.K. Ly, M.J. Patterson, M.M. Maye, G. Liu, C.J. Zhong, Langmuir, 2002, 18, 8255

4 (a) L. Han, J. Luo, N.N. Kariuki, M.M. Maye, V.W. Jones, C.J. Zhong, Chem. Mater, 2003, 15, 29. (b) L. Han, D.R. Daniel, M.M. Maye, C.J. Zhong, Anal. Chem, 2001, 73, 4441. (c) L.B. Israel, N.N. Kariuki, L. Han, M.M. Maye, J. Luo, C.J. Zhong, J. Electroanal. Chem, 2001, 517, 69. (d) N.N. Kariuki, J. Luo, A. Hassan, H.S. Lim, L. Wang, C.J. Zhong, Chem. Mater, 2006, 18, 123. (e) L. Han, M.M. Maye, F.L. Leibowitz, N.K. Ly, CJ. Zhong, J. Mater. Chem, 2001, 11, 1258

5 (a) W.X. Zheng, M.M. Maye, F.L. Leibowitz, C.J. Zhong, Anal. Chem, 2000, 72, 2190. (b) M.M. Maye, J. Luo, L. Han, C.J. Zhong, Nano Lett, 2001, 1, 575. (c) W.X. Zheng, M.M. Maye, F.L. Leibowitz, C.J. Zhong, Analyst, 2000, 125, 17

6 (a) M.M. Maye, H.IS. Lim, J. Luo, Z. Rab, D. Rabinovich, T. Liu, C.J. Zhong, J. Am. Chem. Soc, 2005, 127, 1519. (b) M.M. Maye, J. Luo, H..S. Lim, L. Han, N.N. Kariuki, D. Rabinovich, T. Liu, C.J. Zhong, J. Am. Chem. Soc, 2003, 125, 9906. (c) M.M. Maye, S.C. Chun, L. Han, D. Rabinovich, C.J. Zhong, J. Am. Chem. Soc, 2002, 124, 4958. (d) H.S. Lim, M.M. Maye, J. Luo, C.J. Zhong, J. Phys. Chem. B, 2005, 109, 2578
7 H.S. Lim, J. Ouyang, J. Luo, L. Wang, S. Zhou, C.J. Zhong, Chem. Mater, 2005, 17, 6528

8 H.S. Lim, F. Goroleski, D. Mott, N.N. Kariuki, W. Ip, J. Luo, C.J. Zhong, J. Phys. Chem. B, 2006, 110, 6673

9 (a) S. Link, M.A. El-Sayed, Int. Rev. Phys. Chem, 2000, 19, 409. (b) G.C. Papavassiliou, Prog. Solid St. Chem, 1979, 12, 185

10

$$
T_{\text {film }}=\frac{(1-R)^{2}+4 R \sin ^{2} \psi}{R^{2} \exp (-\alpha d)+\exp (\alpha d)-2 R \cos (\zeta+2 \psi)}
$$

( $T_{\text {firm }}$ : light transmission, R: reflectance at normal incidence, at normal incidence. For details of this equation, see T. Ung, L.M. Liz-Marzan, P. Mulvaney, J. Phys. Chem. B, 2001, 105, 3441

11 J.J. Storhoff, A.A. Lazarides, R.C. Mucic, C.A. Mirkin, R.L. Letsinger, G.C. Schatz, J. Am. Chem. Soc, 2000, 122, 4640

12 C. Sonnichsen, B.M. Reinhard, J. Liphardt, A.P. Alivisatos, Nature Biotechnology, 2005, 23, 741

13 A.J. Haes, R.P. Van Duyne, J. Am. Chem. Soc, 2002, 124, 10596

14 H.W. Yim, L.M. Tran, E.D. Dobbin, D. Rabinovich, L.M. Liable-Sands, C.D. Incarvito, K-C. Lam, A.L. Rheingold, Inorg. Chem, 1999, 38, 2211

15 S.H. Goh, S.Y. Lee, Z.H. Lu, C.H.A. Huan, Macromol. Chem. Phys, 2000, 201, 1037

16 K.C. Grabar, R.G. Freeman, M.B. Hommer, M.J. Natan, Anal. Chem, 1995, 67,735

17 M.M. Maye, L. Han, N.N. Kariuki, N.K. Ly, W-B. Chan, J. Luo, V.W. Jones, C.J. Zhong, Anal. Chim. Acta, 2003, 496, 17

18 M. Alejandro-Arellano, T. Ung, Á. Blanco, P. Mulvaney, L.M. Liz-Marzán, Pure Appl. Chem, 2000, 72, 1

19 (a) F.X. Zhang, L. Han, L.B. Israel, J.G. Daras, M.M. Maye, N.K. Ly, C.. Zhong, Analyst, 2002, 127, 462. (b) H.S. Lim, W. Ip, E. Crew, P. Njoki, D. Mott, C.J. Zhong, Y. Pan, S. Zhou, Langmuir, 2007, 23, 826

20 (a) J. Zhou, C.Q. Sun, K. Pita, Y.L. Lam, Y. Zhou, S.L. Ng, C.H. Kam, L.T. Li, Z.L. Gui, Appl. Phys. Lett, 2001, 78, 661. (b) P.T. Miclea, A.S. Susha, Z. Liang, F. Caruso, C.M.S. Torres, S.G. Romanov, Appl. Phys. Lett, 2004, 84, 3960. (c) Z-Z. Gu, R. Horie, S. Kubo, Y. Yamada, A. Fujishima, O. Sat, Angew. Chem. Int. Ed, 2002, 41, 1154. (d) JJ. Mock, D.R. Smith, S. Schultz, Nano Lett, 2003, 3, 485

21 (a) K.L. Kelly, A.A. Lazarides, G.C. Schatz, Computing in Science and Engineering, 2001, 3, 67. (b) T. Ung, L.M. Liz-Marzan, P. Mulvaney, Colloids and Surfaces A: Physicochemical and Engineering Aspects, 2002, 202, 119. (c) K.E. Peceros, X. Xu, S.R. Bulcock, M.B. Cortie, J. Phys. Chem. B, 2005, 109, 21516

22 L. Han, X. Shi, W. Wu, F.L. Kirk, J. Luo, L. Wang, D. Mott, L. Cousineau, H.S. Lim, S. Lu, C.J. Zhong, Sensors and Actuators B, 2005, 106, 431 